\title{
Optical-resonator-based biosensing systems: current status and future prospects
}

This article was published in the following Dove Press journal:

Nanobiosensors in Disease Diagnosis

24 June 2016

Number of times this article has been viewed

\section{Anis Nurashikin Nordin \\ Department of Electrical and Computer Engineering, International Islamic University Malaysia, Kuala Lumpur, Malaysia}

\begin{abstract}
Optofluidic devices have emerged as a promising label-free method for sensitive detection of biological molecules. Its advantages of rapid analysis, high specificity, and low interactions with samples have made it a popular device for biological and chemical analyses. This mini review describes state-of-the-art optofluidic devices based on optical resonators for biosensing. Advanced optical resonator geometries such as microspheres, microrings, and their integration with fluidics have been described. It is predicted that to meet demands of disease diagnostics for multiple clinically relevant samples, sizes of optical resonators will shrink further and geometries that enable multiplexing and integration with signal processing will be the trend of the future.
\end{abstract}

Keywords: optofluidics, optical resonators, microrings, biosensors, microspheres, multiplexed microrings

\section{Introduction}

Optical sensors have long been popular for analysis of both biological and chemical samples. The use of light, coupled with plasmonics, fluorescence, and Raman scattering have yielded optical sensors capable of detecting concentrations in the femtomolar range. Other than its high sensitivities, optical sensors also allow rapid analysis, high specificity due to specific light-matter interaction, low interactions with samples, and simultaneous detection of multiple analytes via multiplexed systems. ${ }^{1,2}$ The advancement in microfabrication technologies has also led to the miniaturization of optical sensing systems, with bulky flow cytometers and microplate readers transformed into chip-sized waveguides and resonators. ${ }^{3}$ This technology has also enabled the miniaturization of fluidic systems, which are now capable of performing automated particle sorting and separation, cell culturing, and concentration gradient formation for small sample volumes (femtoliters to nanolitres). When coupled with fluids, optics forms a powerful field known as optofluidics, which allows seamless integration of fluid transport with submicron level of analysis.

Both miniaturization and integration of optics with fluidics bring several added advantages when used for biological or chemical sensing. Optical properties such as refractive index (RI), fluorescence, Raman scattering, absorption, and polarization are enhanced in miniaturized systems. The physical structure of optical waveguides or resonators can also be used for biological sample flow, creating compact liquid waveguides. ${ }^{4}$ In some cases, optical forces can also be used to manipulate and trap biological particles, making optofluidic-based biosensing systems both very sensitive and
Department of Electrical and Computer Engineering, Engineering Faculty, International Islamic University Malaysia Jalan Gombak, 53100 Kuala Lumpur, Malaysia

Tel +60 I7 3005043

Email anisnn@iium.edu.my
Nanobiosensors in Disease Diagnosis 2016:5 4I-50

(c) (1) (5) 2016 Nordin. This work is published and licensed by Dove Medical Press Limited. The full terms of this license are available at https://www.dovepress.com/terms.php

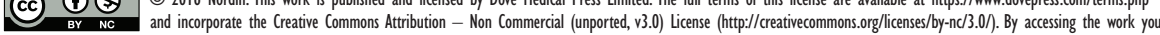
hereby accept the Terms. Non-commercial uses of the work are permitted without any further permission from Dove Medical Press Limited, provided the work is properly attributed. For permission for commercial use of this work, please see paragraphs 4.2 and 5 of our Terms (https://www.dovepress.com/terms.php
Dovepress

http://dx.doi.org/10.2147/NDD.S70385

11 
selective. An important challenge for optofluidic devices is how to achieve maximum interaction between biomolecules and the optical field. ${ }^{4}$

Recent interest in this field of optofluidics has been enormous, with a plethora of biological and chemical sensors being developed. Optical resonators have been reported to be successful in detecting proteins, ${ }^{5}$ cells, ${ }^{6}$ nucleic acids, ${ }^{6,7}$ lectins, ${ }^{8}$ and viruses. ${ }^{9}$ This review will briefly describe the basic principle of operation for optofluidics. Different optical resonator geometries will be described, such as rings, spheres, and discs, with focus on the latest state-of-the-art devices that can be fabricated using microfabrication technology. The biological interactions with these optofluidic sensors will also be described, with emphasis on several recent interesting sensors. The focus of this paper will be more on the advancement in optical resonator geometries for effective biosensing rather than the functionalization methods of surfaces for biosensing techniques.

\section{Optical microsensors}

Optical microsensors utilize light (generated by laser diodes or other light sources) for detection of samples. When the sensor structure is designed such that it confines light within a cavity, creating resonance, it acts as an optical filter, allowing only specific optical frequencies to pass through. Resonant devices that have precise geometric properties such as shape, size, and composition are known as morphologydependent resonances. ${ }^{3}$ The resonant phenomenon is ideal for sensing, as changes in the material within the cavity will also change the RI of the light and also the resonant optical frequency. Change in resonant optical frequency is observable via monitoring of transmitted light intensity. This property makes optical resonators ideal for biosensing, as submicron changes in the microcavity, such as coating of molecular receptors within the cavity, will bring about change in transmitted light intensity. Other applications using microcavities are also possible, where microcavities filled with magnetorestrictive materials can be used as magnetometers, and microcavities with rotating frame of reference can function as gyroscopes.

\section{Resonator geometries}

There are a plethora of different optical resonator geometries, ranging from very simple ones such as spherical, disc, and ring cavities, to more complex ones such as toroidal, tubular, and microbubble, each with its own advantages. We will now explore different resonator geometries, their ease of fabrication, and sensing qualities.

\section{Microsphere}

Microsphere cavities have the simplest optical resonator geometry and are also easy to fabricate. High quality optical fibers are melted using either a hydrogen torch or a carbon dioxide laser beam to form microsized spheres. ${ }^{10,11}$ An important class of resonators which are popular in optics is the whispering-gallery mode (WGM) resonators. WGMs occur within a concave surface as shown in Figure 1A and can be applied both in acoustics and optics. Light traveling within the glass sphere is bounded due to total internal reflection, and the concave structure allows repeated reflections, and neglecting absorption, scattering, and material dispersion, it can continue to travel infinitely. ${ }^{12}$ Extreme sensitivities can be achieved when this mode is applied for sensing, as infinitely repeating reflections can allow multiple interactions with the samples.

When miniaturized, microspheres confine light through total internal reflection, typically light in modes localized along its equator. Microsphere resonances are analogous to photonic atom modes, where massless photons interact with each other so strongly that it appears as if they have mass in them. ${ }^{13,14}$ The trapped light has resonance if an integer number of wavelengths fit on the closed circular optical path. Light is usually coupled from a laser source to the microsphere via an optical waveguide. Resonance is identified from a sharp Lorentzian-shape spectral response obtained at the photodetector. Free-space coupling of light into and from microspheres is not trivial as it is intrisically hindered by its rotational symmetry. ${ }^{15}$ Complex optical and mechanical systems are required to achieve precise alignment of light on the cavity edge. A simpler setup to obtain free-space coupling has been demonstrated by researchers in Spain. ${ }^{16} \mathrm{In}$ this method, an efficient pumping scheme was used to focus laser into the microsphere and to excite $\mathrm{Nd}$ ions. These ions have fluorescent emissions which produce WGMs.

Spheres immersed in liquid have been very successful for biosensing applications and have been reported to be able to detect DNA, streptavadin, thrombin enzyme, and many others. ${ }^{14}$ For effective biological detection, microspheres have to confine light in modes localized along its equator and also exhibit high quality factors (high-Qs) (Figure 1B). Molecules that bind on the surface of the microsphere cause a shift in the resonant frequency as they disrupt the optical field and increase the round trip path length. Evanescent fields or waves occur at boundaries with total internal reflections, as acoustical waves cannot be discontinuous at a boundary. As shown in Figure 1C, evanescent fields occur along the equator line of the microsphere. The evanescent field 

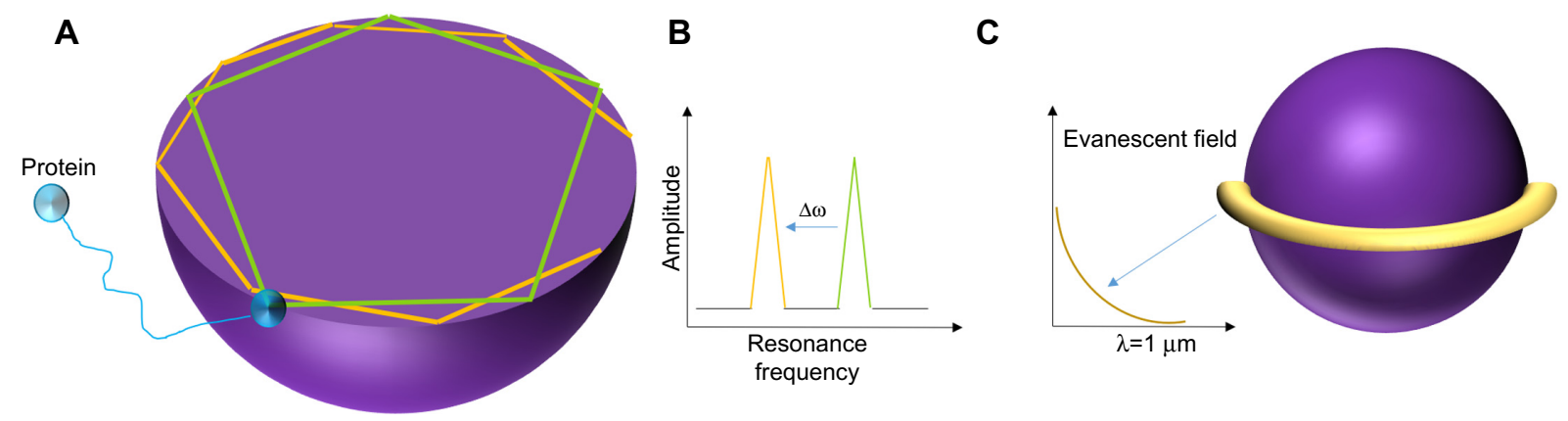

Figure I (A) Optical whispering gallery modes in spheres. (B) Resonant frequency shift observed during binding. (C) Polarization of biomolecules in evanescent field.

decays exponentially without absorption within $\lambda$ distance from the boundary. If the biomolecule binds at the location where the evanescent field strength is high, the molecule becomes polarized. Resonant frequency shifts can then be used to quantify molecule binding events. The shape of the spheres also largely influences the sensitivity of the device. It is interesting to note that perfect spheres cause degeneration of azimuthal modes, whereas slightly imperfect spheres made by melting of fiber tips create a spectrum rich with azimuthal modes with different $m$ numbers. ${ }^{14}$ The observation of transverse electric and transverse magnetic modes in a microsphere can also determine the orientation of the molecules bound on its surface.

Another interesting method of quantifying molecular binding events is via observation of mode-splitting in WGMs. Adsorption of particles in the resonator induces mode-splitting scattering of standing waves. ${ }^{17,18}$ Differential measurement of mode-splitting amount provides results which are more immune to noise compared to resonant-shift-based sensing schemes. ${ }^{19}$ Another advantage of using mode-splitting as the sensing mechanism is that it provides high-resolution measurements, up to the size of a single particle, regardless of the location of the particle in the resonator.

Efforts to enhance the sensitivity of WGM resonators have also been made. An example of such work is explained by Holler, ${ }^{20}$ in his study in which gold nanoparticles were affixed to the resonator's surface to increase the sensitivity of the sensor. The gold nanoparticles act as nanoscopic antennas that boost the electric field within the cavity via increase in the sensor's surface area, thus enhancing the resonator's response to binding events. Using this technique, the researchers have reported the detection of proteins as small as $5 \mathrm{kDa}$, and this technique also has the potential to screen cancer biomarkers that are in the range of $50-300 \mathrm{kDa} .{ }^{20}$ Another interesting approach to increase sensitivity is to use liquid spheres in the form of droplets as WGM resonators. Liquid droplets produce sharp WGM resonances and can serve as both the sample and optical cavity simultaneously. ${ }^{21}$ This allows direct investigation of the chemical composition and detection of nanoparticles in the droplet samples. Using this method, enhanced cavity sensing is done via the intracavity field instead of the evanescent-tail, which is typical in solid microcavities.

An example of application of these microspheres has been demonstrated via detecting the binding kinetics of the streptavidin-biotin pair. ${ }^{22}$ Specific targeting of streptavidin was achieved via immobilization of one-half of the binding pair on the microsphere's sensing surface. Bioactivity of the probe molecule was monitored during the functionalization process by observing the change in the quality factor of the microspheres. The strength of the biotin-streptavidin interaction is approximated via the relationship between the resonant frequency shift and the dissociation constant $\left(k_{\mathrm{d}}\right)$ and association constant $\left(k_{\mathrm{a}}\right)$. The association constants are affected by the fluid flow rate, indicating that there are mass transport limitations hindering the association of streptavidin with immobilized biotin. ${ }^{22}$

Successful detection of nucleic acid hybridization has also been demonstrated via glass microspheres with plasmon enhancements using nanorods. ${ }^{23}$ Monitoring of single-molecule hybridization kinetics was achieved by significantly enhancing the optical field strength on the surface of the microsphere by placing nanorods. These nanorods with volumes in the $\mathrm{nm}^{3}$ range are significantly smaller than the silica microspheres, which have $\sim 30-50 \mu \mathrm{m}$ radius. The placement of these nanorods resulted in sensitivity gains of several thousands, enabling the sensor to detect interaction kinetics between matched and mismatched oligonucleotides as well as octamers. ${ }^{23}$ The usage of plasmonic nanostructures (nanoshells) has also enabled detection of nanoparticles ${ }^{24}$ and 
A

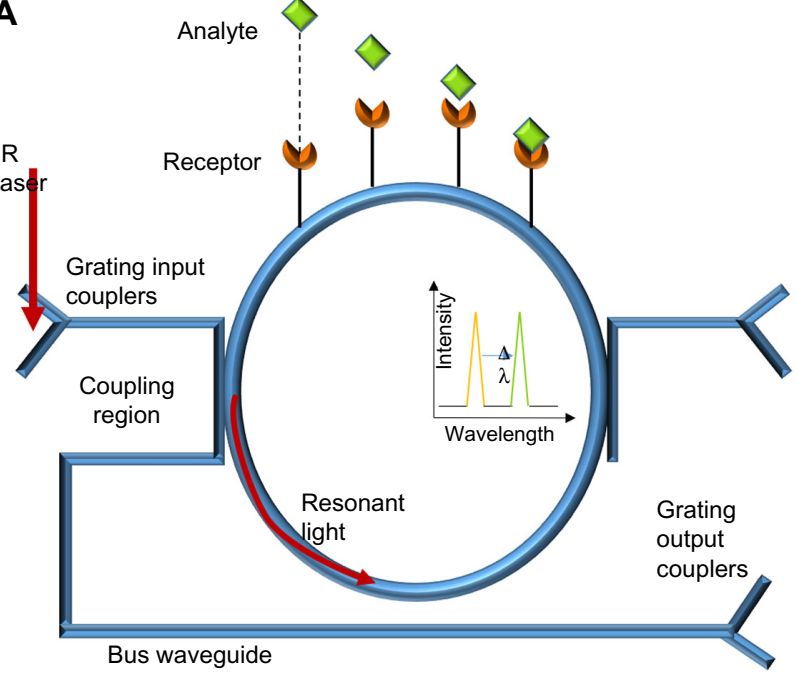

B

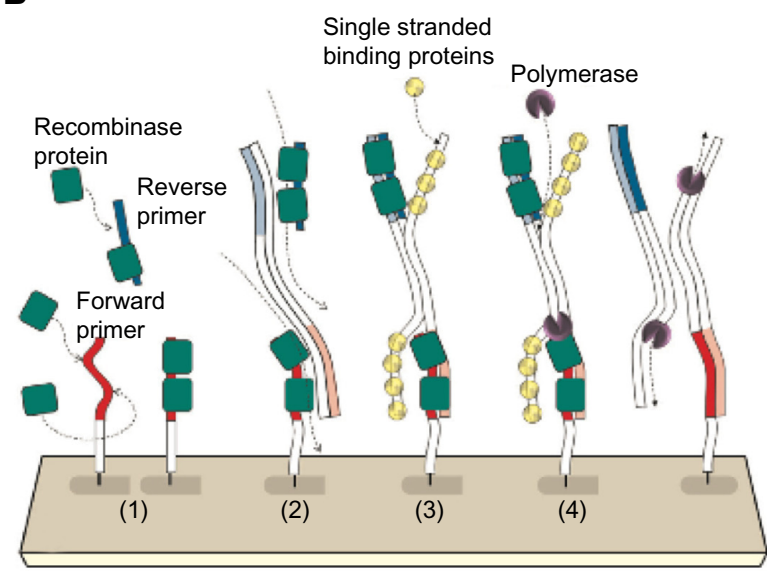

Figure 2 (A) Working principle of a ring resonator. (B) Schematic of solid-phase recombinase polymerase DNA amplification on the resonator. Notes: Reprinted from Biosens Bioelectron, 2015;73:130-137. Sabaté del Río J, Steylaerts T, Henry OYF, et al. Real-time and label-free ring-resonator monitoring of solidphase recombinase polymerase amplification. ${ }^{2}$ @) 2015 with permission from Elsevier, http://www.journals.elsevier.com/biosensors-and-bioelectronics/. Abbreviation: IR, infrared.

the smallest RNA virus MS2.25,26 This technique employs microspheres with nanoplasmonic receptors placed at its equator, providing enhancement of $\sim 70 \times$. Using the reactive sensing principle, nanoparticles entering an evanescent field cause resonance wavelength shifts. These wavelength shifts also cause proportional changes in masses. Microspheres have been previously successful in detecting Influenza A viruses of masses 512 ag. ${ }^{27}$ Plasmonic enhancements, however, can detect MS2 virus with mass of 6 ag since it enhances the intensity of adsorption at the sensing site. Other than viruses, microspheres with plasmonic enhancements have also demonstrated successful detection of cancer protein biomarkers (bovine serum albumin [BSA] and $\mathrm{Tg}$ ) with extremely low masses of 1 and $0.11 \mathrm{ag}$, respectively. ${ }^{20}$

\section{Microring resonators}

While spheres show significant sensitivities and functionalities, reliable fabrication methods for mass production as well as integration with sensing arrays are a bit of a challenge. Twodimensional structures are easier to fabricate using conventional microfabrication methods such as lithography, etching, deposition and standard integrated circuits microfabrication methods such as complementary metal oxide semiconductors. ${ }^{28}$ Ring resonators have been reliably fabricated in the critical coupling conditions with minimum insertion loss, have high-Qs and $>10 \mathrm{~dB}$ extinction ratio. A common structure for optical resonators is rings and discs, which can be fabricated in silica, ${ }^{29}$ silicon, ${ }^{30-32}$ and organic polymers. ${ }^{33,34}$ The design of the planar ring allows confinement of light within the cavity, creating resonance due to total internal reflection. A byproduct of total internal reflection is evanescent waves, which permeate within $\lambda$ distance outside the diameter of the ring. Planar ring resonators have light guided in a solid medium while liquid samples flow near the sensing surface. The biological samples are probed by the evanescent waves, where organic molecules with higher RI are bound to the surface of the ring, while fluids with lower RI (such as water) are displaced.

Figure 2A illustrates a ring resonator that couples infrared light via grating input couplers. The $220 \mathrm{~nm}$ thick ring resonators are fabricated in silicon on silicon-on-insulator wafers. A $5 \mathrm{~nm}$ thick thermal oxide layer was grown on top of the ring resonator structure to create two layers ( $\mathrm{Si}$ and $\mathrm{SiO}_{2}$ ) with different refractive indices. Silicon is transparent to infrared light and acts as a waveguide. Due to the different refractive indices, light is confined to the waveguide and is coupled to the ring-shaped structure. The ring design traps specific wavelengths of light waves in resonance, allowing it to perform $10^{4}-10^{8}$ loops around the ring before it couples out. Interaction with the sample is not only limited by the ring's length but also by the number of times the light waves interact with the sample.

The evanescent field penetrates several tens of nanometers within the perimeter of the ring into the surrounding medium. ${ }^{2}$ Detection of biological samples can be achieved either by changing the RI of the bulk solution (bulk sensing), or by depositing multiple layers on the sensing surface (surface sensing). ${ }^{35}$ Unlike WGM resonators, microrings are single-mode waveguides, which makes it easier to produce 
large free-spectral range with tunable lasers. ${ }^{36}$ Due to its ease of fabrication and since it is based on more mature silicon fabrication technology, the biological applications using ring resonators are more developed. Ring resonators have been used to detect proteins, ${ }^{5}$ cells, ${ }^{6}$ nucleic acids, ${ }^{2,6,7}$ and viruses. ${ }^{9}$

Figure 2B illustrates the schematic representation of solid-phase recombinase polymerase (RPA) for DNA amplification. ${ }^{2}$ The device senses changes in RI on the microring resonator during RPA. Unlike polymerase chain reaction, RPA is an isothermal DNA amplification technique and does not require thermal cycling to cause denaturation of the target and annealing with the primers. Elongation is achieved at a constant and low temperature using enzymes. The microring resonator detects surface amplification of the primers, which are covalently linked to the surface of the resonator. The RPA technique allows rapid direct detection of doublestranded DNA without denaturation and hybridization of the amplification products and uses less reagents. ${ }^{2}$

Another interesting microring resonator setup is described in the work by Kim et al, ${ }^{37}$ which eliminates the use of an expensive and bulky high-resolution wavelength tunable laser to measure resonance wavelength shifts. The system employs two serially cascaded microrings - sensing and trace - each with a separate resonance wavelength $\left(\lambda_{1}, \lambda_{2}\right)$. Before the biological binding event, the difference in resonance peaks is recorded via measurements of its electrical power spectrum. Figure $3 \mathrm{~A}$ shows that the output power is at its maximum if $\lambda_{1}=\lambda_{2}$. The initial resonant difference is recorded as $\lambda_{2}-\lambda_{1}$. Once the binding event occurs, the change in resonant wavelength is monitored as shown in Figure $3 \mathrm{~B}$ via the change in power $(\mathrm{W})$ :

$$
\Delta \mathrm{W}\left(\lambda_{2}\right)=\mathrm{W}_{2}\left(\lambda_{2}^{\prime}-\lambda_{1}\right)-\mathrm{W}_{1}\left(\lambda_{2}-\lambda_{1}\right)
$$

Similar to the concept of differential electronics, differential sensor measurements yield amplified results, making it unnecessary to have precision lasers to measure the wavelength shifts. The double microring system was first verified by measuring the dissociation constant $\left(k_{\mathrm{d}}\right)$ for the well-characterized biotin-streptavidin interaction. ${ }^{37}$ The obtained $k_{\mathrm{d}}$ value measured using the dual-ring resonators was the same order of magnitude as when measured using quartz crystal microbalance and enzyme-linked immunosorbent assay techniques.

Typically, microring resonators require the fluidics to flow in a microchannel parallel to the sensor surface, also known as the flow-over approach. At low concentrations of analytes, this technique is inefficient as the transport rate of the analytes to the surface is very slow. A more effective approach is the flow-through method in which the analyte flows perpendicular to the sensors' surface. ${ }^{35,38}$ High transfer rate of the analytes over the sensor's surface dramatically
A
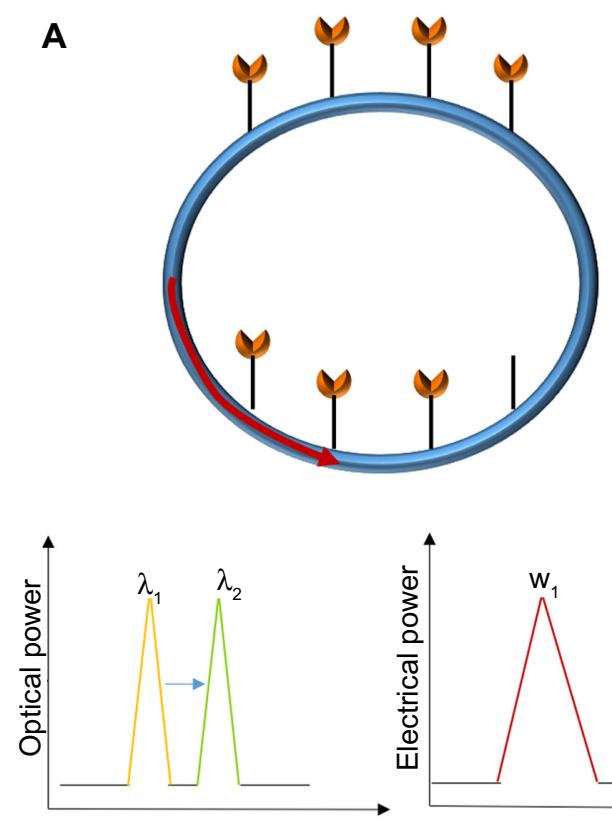

Wavelength, $\lambda$

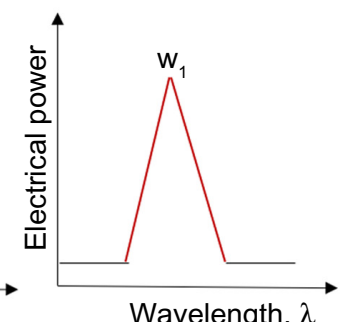

Wavelength, $\lambda$

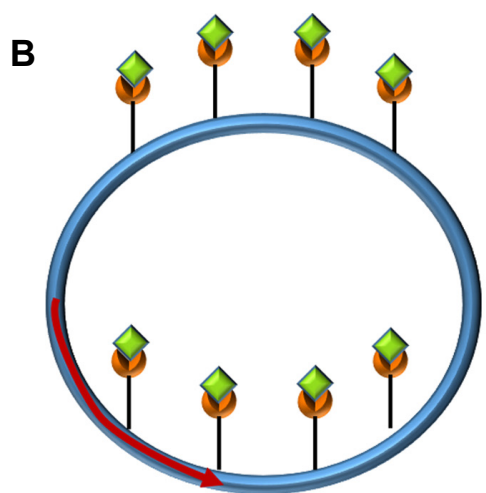

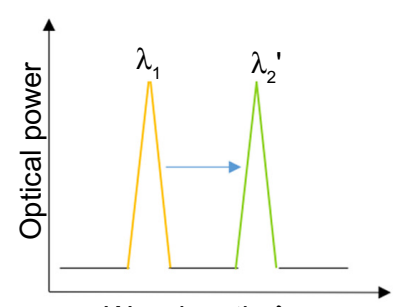

Wavelength, $\lambda$

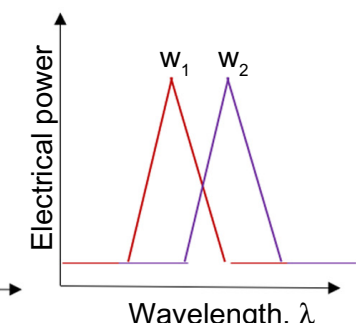

Wavelength, $\lambda$

Figure 3 Dual microring resonator optical system.

Notes: (A) The difference between the tracing and sensor microrings' wavelengths are captured as power $\left(\mathrm{W}_{1}\right)$ before antigen binding. (B) Antigen binding occurs in the sensor microring and the difference in wavelengths are also recorded as power $\left(\mathrm{W}_{2}\right)$. Difference in power $\left(\mathrm{W}_{1}-\mathrm{W}_{2}\right)$ is proportional to the surface density of the polymer layer covering the sensor microring. 
improves the sensor's response time. Figure 4A illustrates the flow-through method employed over a ring resonator for detection of BSA. ${ }^{35}$ The device can detect as low as $20 \mathrm{nM}$ of BSA via measurements of shifts in resonance peaks. Compared to flow-over systems, this flow-through setup shows much faster response times (time taken to increase from $10 \%$ to $90 \%$ of the equilibrium value). As shown in Figure $4 \mathrm{~B}$, the flow-through device requires 4.1 minutes compared to the 14.8 minutes required when using the flow-over device. The measurements were taken at the flow-rate of $6 \mu \mathrm{L} / \mathrm{min}$.

\section{Tubular and liquid core optical ring resonators}

Tubular optical resonators, also known as optofluidic ring resonators (OFRRs), are rolled-up glass microtubes, which are combined with microfluidics for biosensing applications. When light is circulated in the tube, it forms an evanescent field, which can interact with samples within a few nanometers inside and outside the tube. Its sensing mechanism relies on the change in RI of evanescent waves near the surface of the tubular wall. When fluids of different RIs are flowed into the tube, the peak locations of the resonant modes move in order to maintain resonance. ${ }^{39}$ Tubular geometries allow compact combination of fluidics, with sensitivities to optical resonance. Figure 5A(inset) shows the rolled-up glass microtube, which is prepared separately. The microtube is then transferred and fixed on SU-8 sockets, which are placed on a silicon chip (Figure 5B and C). Fabrication of such a device is very complicated, requiring separate fabrication of the microtubes, fixing and transferring of the tubes on a microchip, and finally sealing the entire structure with polydimethylsiloxane to form a microfluidic system. ${ }^{39}$ The sensor has been successful in detecting changes in RIs of deionized water and phosphate-buffered saline (PBS), but it has not been tested for a specific biological application.

Liquid-core optical ring resonators derive their structure from tubular resonators, which are connected to form a ring. ${ }^{14}$ Light coupled into the ring forms WGMs due to internal reflection of light within the ring. ${ }^{29}$ Unlike tubular structures, fluids do not flow within the tube, but instead flow within the core of the ring. Detection is made possible by monitoring the change of the RI of the evanescent field, which is sensitive to the fluid flowing in the core, ${ }^{29}$ as shown in Figure 6. Liquid-core optical ring resonators structures are arguably the most successful structure in biosensing when compared to other structures, having demonstrated their applications in the detection of breast cancer biomarker, ${ }^{40} \mathrm{DNA},{ }^{41}$ messenger RNA (mRNA) ${ }^{42}$ interleukins, ${ }^{43}$ C-reactive proteins, ${ }^{44}$ tumor necrosis, ${ }^{45}$ and antibodies. ${ }^{46}$

An example of such an application is the usage of optofluidic resonators to detect human epidermal growth factor receptor 2 (HER2), a breast cancer biomarker. ${ }^{47}$ Detection and monitoring of HER 2 is important since it is associated with very aggressive types of breast cancer tumors and is related with the high probabilites of cancer relapse. HER2 can be tested in patients' blood samples, eliminating the need of invasive tissue samples. Ring resonator sensing techniques are superior to current label-free detection devices as they have mass detection limits of sub-picogram/millimeter ${ }^{2}{ }^{47}$ Rapid, label-free detection of HER2 extracelllular domain in human serum was successfully carried out using an OFRR by monitoring the change in the effective RI. The OFRR surface is first primed by washing with low concentrations of hydrofluoric acid and ethanol. After baking, dimethyl pimelimidate crosslinkers in PBS are passed through the OFRR. This step is necessary to bind protein G to the aminosilanes. Recombinant protein $G$ helps to orient the HER2 antibodies in optimal positions to capture the breast

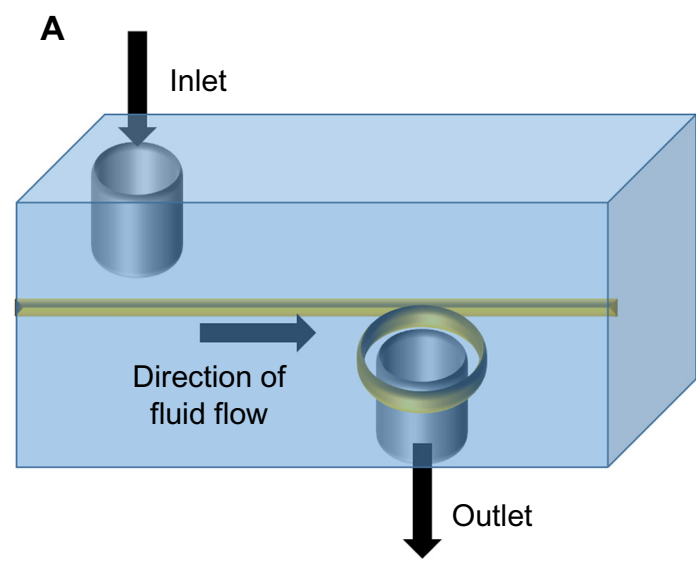

B

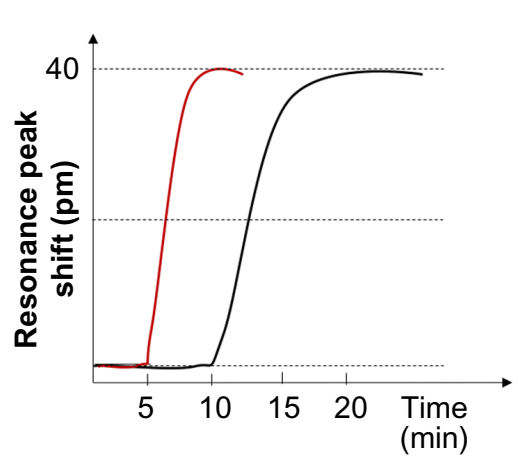

Figure 4 (A) Flow-through microring setup. (B) Comparison of response times using flow-over and flow-through designs. 

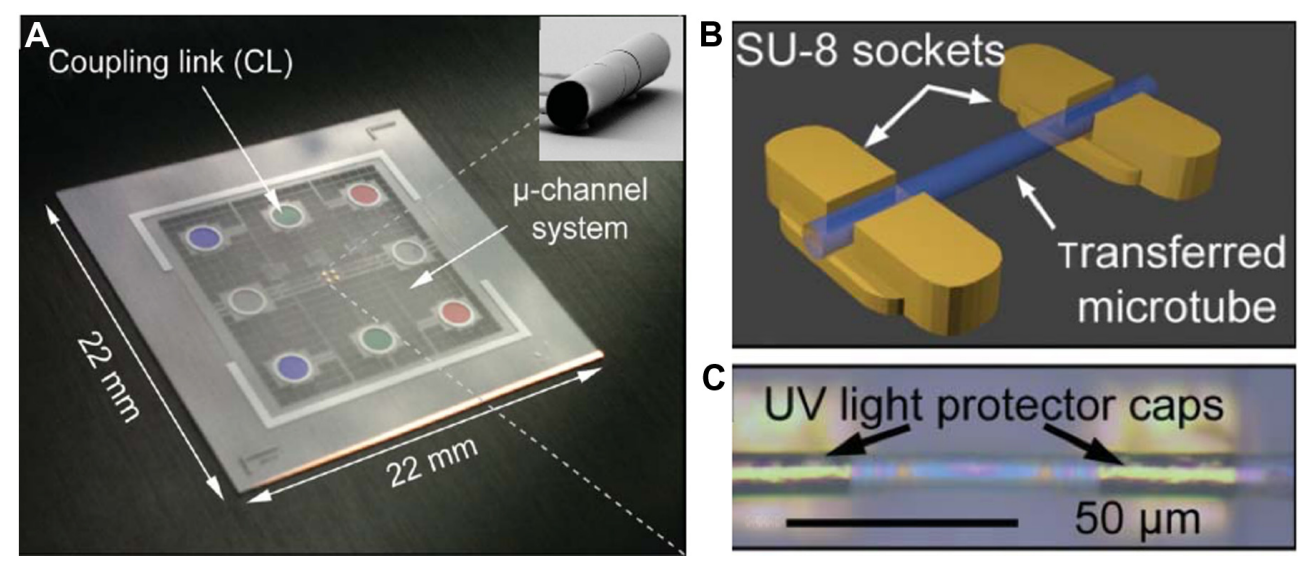

Figure 5 (A) Overview of entire chip with three rolled-up microtubes. (B) Microtube in SU-8 sockets. (C) Optical microtube with ultraviolet light protector caps. Reprinted with permission from. ${ }^{39}$

Note: Reproduced from Harazim SM, Bolaños Quiñones VA, Kiravittaya S, Sanchez S, Schmidt OG. Lab-in-a-tube: on-chip integration of glass optofluidic ring resonators for label-free sensing applications. Lab Chip. 20I2;I2(I5):2649,39 with permission of the Royal Society of Chemistry. Available from http://pubs.rsc.org/en/Content/ ArticleLanding/2012/LC/C2LC40275K\#!divAbstract.

Abbreviation: $\mathrm{CL}$, coupling link.

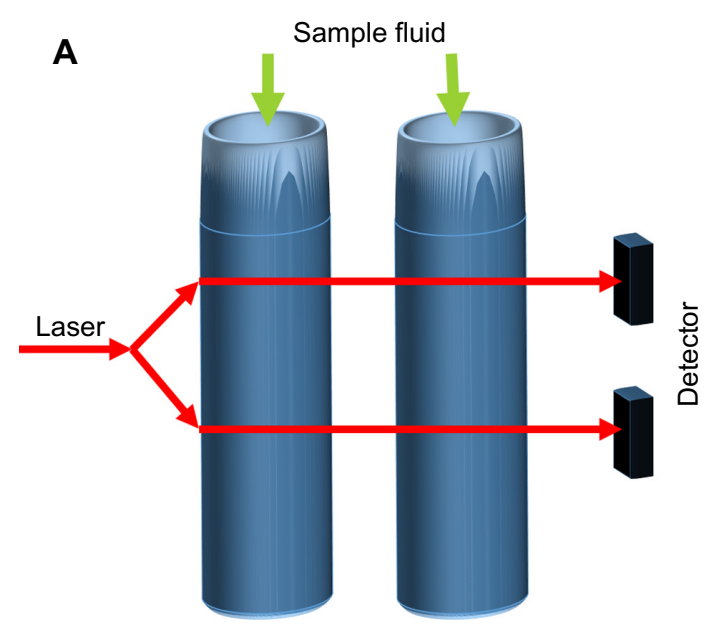

B

Figure 6 (A) Conceptual diagram of liquid core ring resonators (LCORR). (B) Cross-section of LCORR biosensor. Abbreviation: WGM, whispering-gallery mode.

cancer biomarkers. To reduce nonspecific absorptions on the activated surface, a running buffer of casein blocker is flowed on the sensing surface. Concentrations of as low as $20 \mathrm{ng} / \mathrm{mL}$ of HER2 ECD protein were detected via plotting the WGM spectral shifts in a sensorgram over a period of 30 minutes.

Another demonstration of successful implementation of immunoassays using silicon microring resonators was for detection of prostate specific antigen, $\alpha$-fetaprotein, carcinoembryonic antigen, and interleukin- 8 concentrations. ${ }^{43}$ This demonstration was powerful in the sense that it showed that multiplexed analyses can be performed using separate optical microcavities in microfluidic channels without losing sensitivity or measurement precision. The sensing platform is complex, comprising of a sensor array chip, fiber optic splitter, imaging optics, beam splitter, and scanning mirrors. ${ }^{36}$ The usage of a sensor array microchip is advantageous compared to the previous method of using microspheres, ${ }^{48}$ since 32 microrings can be embedded together with its signal routing on a single silicon chip, easing multiplexing signal processing requirements and enabling parallel processing.

Multiplexed array of microrings indeed seem to be the way forward for disease detection using optical resonators. This technique is extremely suitable in clinical settings where sample sizes are limited, assay costs need to be low, and rapid results are required. Other than detection of immunoassays, multiplexed optofluidic rings have also been used to detect microRNAs (miRNAs). miRNAs are a valuable disease biomarker for a number of diseases including 
cancer, ${ }^{49}$ neurodegenerative disorders, ${ }^{50}$ and diabetes. ${ }^{51}$ In a recent work, multiple different miRNAs were simultaneously detected using a modular array of multiplexed silicon photonic microring resonators..$^{42}$ Detection limits were very good; and $\sim 150$ fmol of miRNA was detectable using singlestranded DNA capture probes. The technique was also able to differentiate between single-nucleotide polymorphisms from the let-7 family of miRNAs.

\section{Conclusion and future trends}

Optical sensors have been a preferred biosensing method for decades because of their rapid analysis, high sensitivities, low interactions with samples, high specificity, and capability of detecting several analytes simultaneously. ${ }^{2}$ Recent advances in the field of microfabrication, microfluidics, and photonics have enhanced the rapid progress of optofluidics devices. This work has reviewed several state-of-the-art optical resonators used for biosensing, with the emphasis on the current resonator designs and structures for effective sensing. Microspheres have been reported to successfully detect protein adsorption, thrombin, and viruses. Future work would be on how to further enhance the sensitivities of the sensor. The limit of detection for sensors in general is determined by the active sensing surface. For microspheres, this limits the sensing area to the sphere's equator line. One method to increase surface area is to surround the sphere with gold nanoparticles such that it acts as nanoscopic antennas..$^{20}$ Amplifying the sphere's surface area also amplifies the output signals. Progress in understanding the surface chemistry or functionalization techniques and thus improving accuracy of the microsphere biosensors via increasing the number of accessible binding sites would also be another method of increasing sensitivities. ${ }^{14}$

Although reliable, commercial mass fabrication of microspheres is still very challenging. An alternative design is the use of planar microrings. Due to their compatibility with siliconbased manufacturing techniques, there has been a lot of work done using biosensors based on planar microrings. Optical microring sensors have been successful in detecting mRNAs, prostate specific antigen, and interleukin. ${ }^{14}$ Microring sensor's ease of fabrication allows designers to play around with different arrangements of the microrings, such as placing them in arrays $^{2}$ and differential configurations, ${ }^{37}$ to provide better output signals. The usage of array-based microrings on a single chip paves the way for future applications involving multiplexed analysis of clinically relevant samples. ${ }^{43}$ Complex sensing platforms comprising of the sensing chip, lasers, collimators, and imaging optics have successfully been realized and are capable of running quadruplicate analyses using 20 independent sensors. This method has been successful in detecting five clinically relevant protein biomarkers (carcinoembryonic antigen, prostate specific antigen, $\alpha$-fetoprotein, interleukin- 8 , and tumor necrosis factor- $\alpha$ ) simultaneously. ${ }^{43}$ Ultimately, siliconbased microrings allow on-chip integration with integrated circuits. Although not yet demonstrated, it may be predicted that in the future, complex circuitry can be designed to reduce noise and remove the necessity of costly high-resolution wavelength tunable lasers to measure resonance wavelength shifts.

Effective sensing also requires efficient sample delivery and transport mechanisms. A key problem faced by microringbased biosensors is their slow response times. Typically, ring resonators use flow-over techniques, which require the analyte to traverse the entire loop before a response can be measured. Different designs of fluidic chambers, such as those with flow-over techniques have dramatically improved the sensor's response times. ${ }^{35}$ Optical resonators are also superhydrophobic, making it difficult to concentrate and force biological molecules to adhere to the sensing surface. One option to solve this is using digital microfluidics, which can electrically actuate droplets via electrodes and force fluid flow. ${ }^{52}$ Another method to combat superhydrophobicity is to embed plasmonic nanostructures to improve surface adhesion and cause the solution to precipitate on the surface after evaporation. ${ }^{53}$ Precise manipulation and control of biomolecules can also be achieved using on-chip optical traps. These traps are formed using embedded microheaters placed at the antinodes of a standing wave evanescent field in the nanophotonic waveguide and have demonstrated successful manipulation of DNA molecules. ${ }^{54}$ The recent advances in the development of optofluidic devices have demonstrated the success of proof-of-principle experiments conducted in the lab. Before such devices can be applicable for clinical diagnostics, a stable and reliable fabrication method is required for mass production of these devices and clinical testing.

\section{Disclosure}

The author reports no conflicts of interest in this work.

\section{References}

1. Hunt HK, Armani AM. Label-free biological and chemical sensors. Nanoscale. 2010;2(9):1544.

2. Sabaté del Río J, Steylaerts T, Henry OYF, et al. Real-time and labelfree ring-resonator monitoring of solid-phase recombinase polymerase amplification. Biosens Bioelectron. 2015;73:130-137.

3. Foreman MR, Swaim JD, Vollmer F. Whispering gallery mode sensors. Adv Opt Photonics. 2015;7(2):168.

4. Fluidic vision. Nat Photonics. 2011;5(10):567.

5. Park MK, Kee JS, Quah JY, et al. Label-free aptamer sensor based on silicon microring resonators. Sens Actuators B Chem. 2013; 176:552-559. 
6. Gohring JT, Fan X. Label free detection of CD4+ and CD8+ T cells using the optofluidic ring resonator. Sensors. 2010;10(6):5798-5808.

7. Scheler O, Kindt JT, Qavi AJ, et al. Label-free, multiplexed detection of bacterial tmRNA using silicon photonic microring resonators. Biosens Bioelectron. 2012;36(1):56-61.

8. Kirk JT, Fridley GE, Chamberlain JW, Christensen ED, Hochberg M, Ratner DM. Multiplexed inkjet functionalization of silicon photonic biosensors. Lab Chip. 2011;11(7):1372.

9. Zhu H, White IM, Suter JD, Fan X. Phage-based label-free biomolecule detection in an opto-fluidic ring resonator. Biosens Bioelectron. 2008;24(3):461-466.

10. Collot L, Lefèvre-Seguin V, Brune M, Raimond JM, Haroche S. Very high- $Q$ whispering-gallery mode resonances observed on fused silica microspheres. Europhys Lett EPL. 1993;23(5):327-334.

11. Fan X, White IM. Optofluidic microsystems for chemical and biological analysis. Nat Photonics. 2011;5(10):591-597.

12. Strutt JW. The problem of the whispering gallery. Philos Mag. 1910;20:1001-1004

13. Arnold S. Microspheres, photonic atoms and the physics of nothing light can become trapped within tiny, transparent spheres. The surprising properties that result may turn "microsphere photonics" into an important new technology. Am Sci. 2001;89(5):414-421.

14. Vollmer F, Yang L. Review label-free detection with high-Q microcavities: a review of biosensing mechanisms for integrated devices. Nanophotonics. 2012;1(3-4):267-291.

15. Zhu J, Özdemir ŞK, Yilmaz H, et al. Interfacing whispering-gallery microresonators and free space light with cavity enhanced Rayleigh scattering. Sci Rep. 2014;4:6396.

16. Martín LL, Haro-González P, Martín IR, et al. Whispering-gallery modes in glass microspheres: optimization of pumping in a modified confocal microscope. Opt Lett. 2011;36(5):615.

17. Mazzei A, Götzinger S, Menezes Lde S, Zumofen G, Benson O, Sandoghdar V. Controlled coupling of counterpropagating whisperinggallery modes by a single Rayleigh scatterer: a classical problem in a quantum optical light. Phys Rev Lett. 2007;99(17):173603.

18. Gorodetsky ML, Pryamikov AD, Ilchenko VS. Rayleigh scattering in high-Q microspheres. J Opt Soc Am B. 2000;17(6):1051.

19. Zhu J, Ozdemir SK, Xiao Y-F, et al. On-chip single nanoparticle detection and sizing by mode splitting in an ultrahigh-Q microresonator. Nat Photonics. 2010;4(1):46-49.

20. Holler S. Label-free single cancer marker protein detection using a nanoplasmonic-photonic hybrid whispering gallery mode biosensor. SPIE Sens Technol Appl. 2014;911217.

21. Avino S, Krause A, Zullo R, et al. Direct sensing in liquids using whispering-gallery-mode droplet resonators. Adv Opt Mater. 2014;2(12):1155-1159.

22. Soteropulos CE, Hunt HK, Armani AM. Determination of binding kinetics using whispering gallery mode microcavities. Appl Phys Lett. 2011;99(10):103703

23. Baaske MD, Foreman MR, Vollmer F. Single-molecule nucleic acid interactions monitored on a label-free microcavity biosensor platform. Nat Nanotechnol. 2014;9(11):933-939.

24. Shopova SI, Rajmangal R, Holler S, Arnold S. Plasmonic enhancement of a whispering-gallery-mode biosensor for single nanoparticle detection. Appl Phys Lett. 2011;98(24):243104.

25. Dantham VR, Holler S, Kolchenko V, Wan Z, Arnold S. Taking whispering gallery-mode single virus detection and sizing to the limit. Appl Phys Lett. 2012;101(4):043704.

26. Kuzmanovic DA, Elashvili I, Wick C, O'Connell C, Krueger S. Bacteriophage MS2: molecular weight and spatial distribution of the protein and RNA components by small-angle neutron scattering and virus counting. Structure. 2003;11(11):1339-1348.

27. Vollmer F, Arnold S, Keng D. Single virus detection from the reactive shift of a whispering-gallery mode. Proc Natl Acad Sci U S A. 2008; 105(52):20701-20704.

28. Spearing SM. Materials issues in microelectromechanical systems (MEMS). Acta Mater. 2000;48(1):179-196.
29. White IM, Oveys H, Fan X. Liquid-core optical ring-resonator sensors. Opt Lett. 2006;31(9):1319.

30. Bogaerts W, De Heyn P, Van Vaerenbergh T, et al. Silicon microring resonators. Laser Photonics Rev. 2012;6(1):47-73.

31. Rodriguez GA, Hu S, Weiss SM. Porous silicon ring resonator for compact, high sensitivity biosensing applications. Opt Express. 2015;23(6):7111.

32. Iqbal M, Burlingame R, Romero R, Wang A, Grove T, Gleeson M. Silicon photonic micro-ring resonators for drug screening and kinetic analysis. In: Fang Y, editor. Label-Free Biosensor Methods in Drug Discovery. Methods in Pharmacology and Toxicology. New York, NY: Springer; 2015:133-153.

33. Rowland KJ, François A, Hoffmann P, Monro TM. Fluorescent polymer coated capillaries as optofluidic refractometric sensors. Opt Express. 2013;21(9):11492.

34. Wang L, Ren J, Han X, et al. A label-free optical biosensor built on a low-cost polymer platform. IEEE Photonics J. 2012;4(3):920-930.

35. Grimaldi IA, Testa G, Bernini R. Flow through ring resonator sensing platform. RSC Adv. 2015;5(86):70156-70162.

36. Iqbal M, Gleeson MA, Spaugh B, et al. Label-free biosensor arrays based on silicon ring resonators and high-speed optical scanning instrumentation. IEEE J Sel Top Quantum Electron. 2010;16(3):654-661.

37. Kim KW, Song J, Kee JS, Liu Q, Lo G-Q, Park MK. Label-free biosensor based on an electrical tracing-assisted silicon microring resonator with a low-cost broadband source. Biosens Bioelectron. 2013;46: 15-21.

38. Huang M, Galarreta BC, Cetin AE, Altug H. Actively transporting virus like analytes with optofluidics for rapid and ultrasensitive biodetection. Lab Chip. 2013;13(24):4841.

39. Harazim SM, Bolaños Quiñones VA, Kiravittaya S, Sanchez S, Schmidt OG. Lab-in-a-tube: on-chip integration of glass optofluidic ring resonators for label-free sensing applications. Lab Chip. 2012; 12(15):2649.

40. Hongying Zhu PSD. Rapid and label-free detection of breast cancer biomarker CA15-3 in clinical human serum samples with optofluidic ring resonator sensors. Anal Chem. 2009;81(24):9858-9865.

41. Qavi AJ, Kindt JT, Gleeson MA, Bailey RC. Anti-DNA:RNA antibodies and silicon photonic microring resonators: increased sensitivity for multiplexed microRNA detection. Anal Chem. 2011;83(15): 5949-5956.

42. Qavi AJ, Bailey RC. Multiplexed detection and label-free quantitation of micrornas using arrays of silicon photonic microring resonators. Angew Chem Int Ed. 2010;49(27):4608-4611.

43. Washburn AL, Luchansky MS, Bowman AL, Bailey RC. Quantitative, label-free detection of five protein biomarkers using multiplexed arrays of silicon photonic microring resonators. Anal Chem. 2010;82(1): 69-72.

44. Luchansky MS, Washburn AL, McClellan MS, Bailey RC. Sensitive onchip detection of a protein biomarker in human serum and plasma over an extended dynamic range using silicon photonic microring resonators and sub-micron beads. Lab Chip. 2011;11(12):2042-2044.

45. Luchansky MS, Bailey RC. Rapid, multiparameter profiling of cellular secretion using silicon photonic microring resonator arrays. J Am Chem Soc. 2011;133(50):20500-20506.

46. De Vos K, Girones J, Claes T, et al. Multiplexed antibody detection with an array of silicon-on-insulator microring resonators. IEEE Photonics J. 2009;1(4):225-235.

47. Gohring JT, Dale PS, Fan X. Detection of HER2 breast cancer biomarker using the opto-fluidic ring resonator biosensor. Sens Actuators B Chem. 2010;146(1):226-230.

48. Vollmer F, Arnold S, Braun D, Teraoka I, Libchaber A. Multiplexed DNA Quantification by Spectroscopic Shift of Two Microsphere Cavities. Biophys J. 2003;85(3):1974-1979.

49. Bartels CL, Tsongalis GJ. MicroRNAs: novel biomarkers for human cancer. Clin Chem. 2009;55(4):623-631.

50. Hébert SS, Strooper BD. miRNAs in neurodegeneration. Science. 2007;317(5842):1179-1180. 
51. Walker MD. Role of MicroRNA in pancreatic $\beta$-cells where more is less. Diabetes. 2008;57(10):2567-2568.

52. Luan L, Royal MW, Evans R, Fair RB, Jokerst NM. Chip scale optical microresonator sensors integrated with embedded thin film photodetectors on electrowetting digital microfluidics platforms. IEEE Sens $J$. 2012;12(6):1794-1800.
53. De Angelis F, Gentile F, Mecarini F, et al. Breaking the diffusion limit with super-hydrophobic delivery of molecules to plasmonic nanofocusing SERS structures. Nat Photonics. 2011;5(11):682-687.

54. Soltani M, Lin J, Forties RA, et al. Nanophotonic trapping for precise manipulation of biomolecular arrays. Nat Nanotechnol. 2014;9(6): $448-452$.

\section{Publish your work in this journal}

Nanobiosensors in Disease Diagnosis is an international, peer-reviewed, open access journal publishing original research, reports, reviews and commentaries including but not confined to: Diagnosis of diseases including cancer, cardiovascular, infectious diseases; Molecular modeling in diagnosis; Enzyme and membrane technologies; and quantum dot fluorescence technologies for monitoring toxins and pathogens. The manuscript management system is completely online and includes a very quick and fair peer-review system, which is all easy to use. Visit http://www.dovepress.com/testimonials.php to read real quotes from published authors.

Submit your manuscript here: http://www.dovepress.com/nanobiosensors-in-disease-diagnosis-journa 